\title{
Improving communication in the South African healthcare context
}

\author{
M G Matthews, MB ChB, DOH, MPH; J M van Wyk, BSc Ed, BEd, MEd, PhD \\ Clinical and Professional Practice, School of Clinical Medicine, Nelson R Mandela School of Medicine, College of Health Sciences, University of KwaZulu-Natal, \\ Durban, South Africa
}

Corresponding author: M G Matthews (matthewsm@ukzn.ac.za)

\begin{abstract}
Background. The teaching of communication is an essential component of health professions curricula internationally and in South Africa (SA) for its benefits to healthcare. The teaching, however, remains concentrated in certain disciplines, resulting in an incoherent approach. SA medical schools have incorporated international guidelines into their curricula, but limited research is available on the suitability of amended guidelines for local use. Research in different sociocultural contexts has shown benefits in redefining guidelines for suitability for local contexts.

Objective. To explore suggestions of participants for improving communication teaching and learning in the KwaZulu-Natal (KZN) healthcare context. Methods. Participants, including medical students, urban- and rural-based clinical educators, and Department of Health employees, were purposively sampled. Focus group discussions and semi-structured interviews were conducted. The data were analysed for emergent themes to make recommendations for improved teaching.

Results. Recommendations to improve communication teaching and learning at institutional level included developing isiZulu language and intercultural communication and other aspects of the curriculum. At an individual level, emphasis should be placed on patient-centred care and appropriate professional behaviours, as the 'hidden curriculum' was a noticeable and powerful influence.

Conclusion. Teaching and modelling good communication in clinical skills and clinical teaching should be integrated and complementary. Students require a good grounding in communicating in isiZulu and improved cultural competence. A unified approach that is reached by consensus and tailored to the KZN context is recommended.
\end{abstract}

Afr J Health Professions Educ 2018;10(4):194-198. DOI:10.7196/AJHPE.2018.v10i4.1000

The communicator role and other professional competencies for medical students have been emphasised internationally and in South Africa (SA) as important for healthcare outcomes. ${ }^{[1]}$ While various SA medical schools have adopted aspects of international guidelines for undergraduate health professions curricula, ${ }^{[2,3]}$ there is still limited research on the use of guidelines in the national or regional SA context.

Internationally, there has been growing consensus on principles for inclusion in communication teaching in undergraduate programmes. These principles include attention to faculty development, fostering personal and professional growth, emphasising a patient-centred approach, consistency between communication skills teaching and clinical teaching, implementing a coherent framework and reliable assessments and evaluation of programmes. ${ }^{[4-6]}$ Experts in European countries have developed a Health Professions Core Communication Curriculum (HPCCC) to standardise undergraduate health professions education. ${ }^{[7]}$ The core objectives focus on:

- communication with patients

- intra- and interpersonal communication to foster and develop selfreflection and professionalism

- communication in healthcare teams.

Recent research in communication, while acknowledging the importance of core objectives, has questioned the validity of international guidelines in different sociocultural contexts. Attempts were made to reach consensus on which core principles to include in curricula to ensure their relevance and appropriateness to each context. ${ }^{[8-10]}$ For example, in Indonesia, Claramita and Susilo ${ }^{[9]}$ reported on the strong hierarchical and communal culture that influences the doctor-patient interaction and choices relating to the health of individuals. In that context they recommended the use of a simplified guideline, i.e. the 'Greet-Invite and Discuss' guide to communication to accommodate the cultural characteristics of Southeast Asian patients. ${ }^{[10]}$ De Leonardo et al. ${ }^{[8]}$ similarly recommended that consensus be reached on communication training among Spanish and Portuguese speakers. The development of consensus statements has therefore become necessary owing to regional and cultural practices and language, and the effects of trying to deliver efficient care within overburdened healthcare systems that are often under severe consultation time constraints. ${ }^{[10]}$ It is argued that tailored guidelines for communication that consider the specific contexts of the patients and their caregivers are more user friendly and thus more likely to be adopted in everyday use.

Educating students to function effectively in the multilingual and multicultural SA healthcare context is challenging. In KwaZulu-Natal (KZN) Province, $77.8 \%$ of patients are isiZulu speaking. ${ }^{[1]}$ The students in this context, even those from different language and cultural backgrounds, are expected to become competent in addressing the needs of patients. In health professions education, recommendations have been made for transformation of education to strengthen connections between the education and health systems ${ }^{[12]}$ and for an increased emphasis on interprofessional and collaborative practice. ${ }^{[13]}$ The practical implementation, however, has been challenging, given the increased student intakes and resource limitations. In the current programme, medical students are taught isiZulu in a first-year module, and a patient-centred approach to communication in the disciplines of family and behavioural medicine and in the context of clinical skills. ${ }^{[14]}$ The core 
competencies (including the communicator role) are introduced in the first year, and practical sessions with simulated patients continue throughout the second and third years. The importance of communication (and of language and culture in communication) is revisited in the rural health rotation in the final year. However, the manner in which students communicate with patients and other health professionals in practice is frequently modelled on behaviours observed in the clinical disciplines, ${ }^{[15]}$ a phenomenon generally referred to as the "hidden curriculum. ${ }^{\text {' }}{ }^{[16]}$ Students find it challenging to use a patient-centred approach in busy clinical environments, where the emphasis is on biomedical priorities. This study was therefore conducted to explore the suggestions of participants (local students and staff of the University of KZN and the Department of Health (DoH)) to improve communication teaching and learning and the quality of care and health outcomes of patients.

\section{Methods}

An exploratory, qualitative case study was conducted at the medical school, using focus group discussions (FGDs) and semi-structured interviews (SSIs), in 2015 - 2016. The study population consisted of students, educators and participants from the DoH. Participants were selected purposively to represent the educational and service platforms. For the student group, a final-year student was requested to assemble a diverse group of peers. For the educator groups, an invitation was sent to discipline heads and year or module co-ordinators with knowledge of curriculum content. For DoH participants, staff from hospitals where students complete in-service learning were invited to participate.

The final study sample consisted of:

- Final-year students (FGD 1, $n=5$ ).

- Educators from the schools that teach in the medical programme, viz. School of Clinical Medicine (FGD 2, n=9) and the Schools of Nursing and Public Health and Laboratory Medicine and Medical Sciences (FGD 3, $n=7)$. The total in the educator group was therefore 16 .
- Three DoH participants comprised a rural family practitioner (SSI 1), a rural hospital manager (SSI 2), and a DoH hospital-based researcher (SSI 3).

The participants in the three groups were diverse in gender, language and religious and cultural beliefs (Table 1).

Participants were informed of the study, and written individual consent and permission for audio-recording were obtained. Participants were assured of anonymity. FGDs and SSIs were conducted by the researcher, with an assistant to take notes in the groups. A set of questions was used to initiate the discussions and elicit participants' suggestions. Students had completed their final-year examinations and were aware that there could be no negative consequences related to any expressed views. The duration of the FGDs was 60 - 90 minutes, and that of the SSIs 35 - 45 minutes. Audio-recorded data were transcribed and checked for accuracy by the researchers. Data were analysed by two researchers (a clinician and an educator) for themes related to the study objective, using a social constructionist approach. The themes were reviewed and refined into the main themes at institutional and individual level. ${ }^{[17]}$ Trustworthiness was established through several methods to ensure rigour, ${ }^{[18]}$ including triangulation of the data for multiple perspectives, and the use of various sources (FGDs and SSIs). Additional methods included summarising content and true member checking, and keeping records of the data analysis process as evidence of how the data were generated. Data were reported anonymously, stating only the first language (L1) of the participant.

Gatekeeper permission and ethical approval (ref. no. HSS1633/014) were obtained from the Humanities and Social Sciences ethics committee, University of KZN.

\section{Results and discussion}

This study explored suggestions of participants to improve undergraduate communication teaching and learning. The main themes identified to improve communication teaching on educational and service platforms related to suggestions for implementation at the institutional or individual level (Table 2).

Table 1. Demographic data of participants

\begin{tabular}{|c|c|c|c|}
\hline Variable & $\begin{array}{l}\text { Medical students, } \\
n=5\end{array}$ & $\begin{array}{l}\text { Educators, } \\
n=16\end{array}$ & $\begin{array}{l}\text { Department of Health, } \\
n=3\end{array}$ \\
\hline \multicolumn{4}{|l|}{ Gender } \\
\hline Female & 2 & 10 & 3 \\
\hline Male & 3 & 6 & - \\
\hline \multicolumn{4}{|l|}{ First language } \\
\hline English & 3 & 14 & 2 \\
\hline isiZulu & 1 & 2 & - \\
\hline isiXhosa & 1 & - & - \\
\hline Sesotho & - & - & 1 \\
\hline \multicolumn{4}{|l|}{ Religion } \\
\hline Christianity & 4 & 8 & 3 \\
\hline Hinduism & 1 & 6 & - \\
\hline Islam & - & 2 & - \\
\hline \multicolumn{4}{|l|}{ Academic rank } \\
\hline Associate professor* & $\mathrm{n} / \mathrm{a}$ & 1 & $\mathrm{n} / \mathrm{a}$ \\
\hline Senior lecturer ${ }^{*}$ & $\mathrm{n} / \mathrm{a}$ & 9 & $\mathrm{n} / \mathrm{a}$ \\
\hline Lecturer & $\mathrm{n} / \mathrm{a}$ & 5 & $\mathrm{n} / \mathrm{a}$ \\
\hline Principal programme officer ${ }^{\dagger}$ & $\mathrm{n} / \mathrm{a}$ & 1 & $\mathrm{n} / \mathrm{a}$ \\
\hline
\end{tabular}




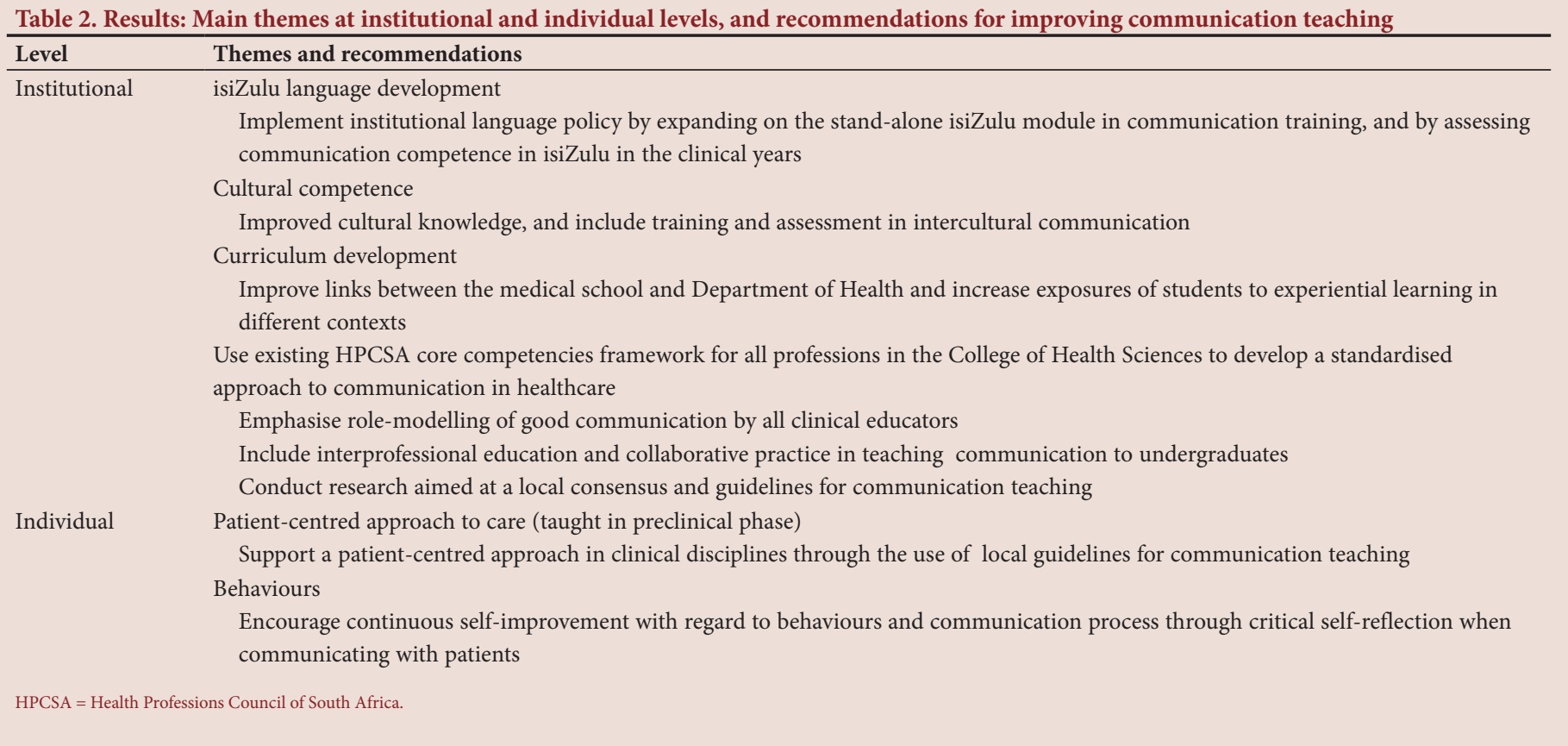

These have been discussed below and linked to recommendations to improve the teaching of communication.

At institutional level, the themes primarily emphasised the necessity for isiZulu language development and matters related to cultural competence and curriculum development. Confirming the findings of previous SA studies, ${ }^{[19,20]}$ a major concern of participants was related to challenges of language barriers between doctors or health professions students and patients. In spite of concerns from some participants regarding professional mobility within the country, participants in all the groups recommended that isiZulu language teaching be improved. They verbalised that current initiatives are inadequate to enable students to communicate effectively with their isiZulu-speaking patients:

'I think isiZulu is central in this province where we have a high percentage of people whose first language is isiZulu.' (FGD 3, No. 2, L1 English)

The second theme at institutional level related to a greater understanding of cultural diversity and developing cultural competence of students. Training in cultural competence has assumed great importance and has been linked to better health outcomes and improved quality of care: ${ }^{[21,22]}$

'I think there is one area we don't foreground adequately and that is cross-cultural communication.' (FGD 3, No. 6, L1 English)

'Maybe the clinical picture is not making sense, especially in children, with enemas and the like. Ask the question this way, "When did you last see the traditional healer?" instead of saying, "Did you go to a traditional healer?"' (SSI 2, L1 Sesotho)

Concern was expressed not only with regard to isiZulu culture, but also to other cultures; for example, an educator asked:

'[Regarding] the students that come from rural areas here, how much support do we give them to engage with Indian culture or Muslim culture? For them it is as much of a cultural divide and we do not give any language or tools on how to ask the question or how to engage, to understand rituals.' (FGD 3, No. 6, L1 English)

Relating to these two themes, participants advocated strongly for isiZulu to be developed as a clinical competency, with greater emphasis on understanding cultural influences in health:

'Learning Zulu is extremely valuable.' (SSI 1, L1 English)

'We have to adapt to any environment and to any cultural setting.' (FGD 2, No. 7, L1 English)

For students to achieve language and cultural competence, suggestions were made to include isiZulu teaching and assessments in a vertical manner throughout the curriculum:

'Maybe every second year have an exam (in isiZulu).' (FGD 1, No. 3, L1 English)

Suggestions for improving cultural competence included:

'You can teach general principles on ... different cultures and that you need to be aware of it.' (FGD 1, No. 1, L1 English)

'Where they call cultural-based people [e.g. sangoma, ${ }^{*}$ nyanga ${ }^{*}$ to come and interact with the actual doctors at the hospital ... and have some discussions around this.' (FGD 1, No. 4, L1 isiXhosa) [ ${ }^{*}$ traditional healers in isiZulu culture]

Mention was made of the introduction of interpreters and cultural brokers, but this would seem an unlikely eventuality given the prevailing economic limitations in health, thus strengthening the imperative for improving students' familiarity with the appropriate terms for common culture-bound syndromes and traditional medicines. ${ }^{[23]}$

The third theme at institutional level related to curriculum development, including the use of a standard approach to communication, and teamwork 
by health professionals on the educational and service platforms, as recommended in most international guidelines: $:^{[4,7]}$

'People talk a lot about the hidden curriculum. Maybe we should be asking how is that communicated and what does it communicate and then what strategy should be put in place to address, in a more overt way, those particular issues?' (FGD 3, No. 4, L1 English)

'The medical student or doctor needs to feel part of a team and not necessarily above everyone else in the healthcare arena ... I think that might be an issue of arrogance, and not relating to everybody as team members.' (SSI 3, L1 English)

'... we have nurses who help us interpret ... and clinical associates.' (SSI 2, L1 Sesotho)

'If you have a problem that you want to solve, you know you have to be interacting with the people and communicating ... we need a social worker ... a dietician ... and somebody who is going to the community to trace that patient.' (FGD 2, No. 9, L1 isiZulu)

Educators and students confirmed the importance of role-modelling. They mentioned that communication skills were not being reinforced in the clinical setting owing to challenges related to the burden of disease:

'Whereas we can't focus on communication as our number one priority is HIV.' (FGD1, No. 4, L1 isiXhosa)

Students described negative role-modelling, especially at a higher level of care in an urban context:

'I think that changing the mindset [of the institution] ... I know it's difficult ... I think just to reiterate the importance of all this [communication] to them, because they become our role models.' (FGD 1, No. 3, L1 English)

Participants from the specialist disciplines confirmed the practice of negative role-modelling:

'Although we expect students to be able to communicate ... I am not sure it is the formal way in which we role play because that is absent in our teaching.' (FGD 2, No. 1, L1 English)

'Generally speaking, our teaching and our doing are different.' (FGD 3, No. 8, L1 English)

Only one student described a positive role-modelling experience at a rural teaching site, which highlights the importance of the learning environment ${ }^{[16]}$ and of role models ${ }^{[24]}$ as influential factors in health professions training:

'That was a common theme [understanding the patient's perspective] throughout the rural hospital in that most of the doctors I worked with were very positive influences and very positive role models.' (FGD 1, No. 3, L1 English)

The themes identified at individual level were the use of a patient-centred approach and the demonstration of appropriate behaviours. Students' comments suggested that they were motivated to use the patient-centred approach:

'I always ask the patients, "How do you feel about it?" Even though you don't really tell the consultant because they don't really want to know but you do still wonder and you still ask the patient's opinion because you think it's a scary situation. They don't know what's going on and the sad thing is that the majority of the time the patients know very little about their condition, because on the ward round nobody says anything to the patient, except, "Are you okay?"' (FGD 1, No. 5, L1 isiZulu)

However, evidence from students and educators suggested limited adoption of the patient-centred approach in practice, with a clinical educator stating: 'If you look at the doctors, we get the information and we can put it together, but we still don't understand where the patient comes from and what he is feeling.' (FGD 2, No. 8, L1 English)

This supports the findings of a previous study at this institution, ${ }^{[15]}$ which showed limited adoption of a patient-centred approach in a major clinical discipline. These observations re-emphasise the need for constant attention to be paid to the powerful influence on students' learning of the hidden curriculum. ${ }^{[16]}$

Participants discussed undesirable behaviours of health professionals, and made recommendations for behaviours that demonstrated respect and empathy for patients:

'Like sisters [nurses] aren't sensitive about patients' issues, but I find myself, always if I'm going to ask a sensitive question ... I'm consciously aware of the fact that this is a sensitive issue.' (FGD 1, No. 5, L1 isiZulu) 'Respect is the word. If we can teach our students respect, you can teach them so much else, even communication and empathy' (FGD 2, No. 1, L1 English)

'Communication goes beyond the words and beyond the clinical diagnosis. So for the medical schools to create a sense of empathy between the health practitioner and the patient, and a sense of connectedness between the health practitioner and the patient, I would think that you would do that, not by didactic teaching, but by giving the students more experiential learning in the field, where they can see, first hand, how patients may live, but that has to go hand-in-hand with clinical excellence.' (SSI 3, L1 English)

These findings support global initiatives to enhance core values and communication in healthcare, as embraced in the international charter for human values in healthcare. ${ }^{[25]}$ This charter articulates the role of skilled communication in enacting these values, with an emphasis on compassion and respect, and commitment to ethical practice, excellence and justice.

\section{Recommendations}

The findings of this study support the implementation of the institutional language policy, as students require a good grounding in communicating in isiZulu. This could be achieved by expanding on the stand-alone isiZulu module, and by integrating teaching and assessment of communication competence in isiZulu in the clinical years. Students' cultural knowledge and skills in intercultural communication should be included in student evaluations. Other recommendations are to further improve links between the medical school and the $\mathrm{DoH}$ and provide more experiential learning in a clinical context. An interprofessional approach to teaching communication across colleges of health sciences would be beneficial to promote consistency between communication skills and clinical teaching. Good role-modelling of communication and professional behaviours by clinical educators in urban and rural contexts is essential and could be included in performance indicators of staff. 
The findings support the use of a patient-centred approach in communication that considers the needs of individual patients and respects differences, whether related to gender, age, language, culture, religion or other beliefs. Many of the recommendations closely reflect the philosophical principles included in objectives on patient perspectives and health beliefs in the HPCCC: $:^{[7]}$

- 'Considers the somatic, mental, social, gender, cultural, ethical and spiritual elements ... and perceives divergences between own values and norms and the patient's.

- 'Responds to the patient's health beliefs and theories of illness and contrasts and integrates these into own theories as a health care professional.'

The inference, then, is that problems with transferring good communication skills in the KZN clinical environment relate less to policies and principles than to praxis. It is therefore suggested that consensus be reached on the use of an explicit patient-centred approach, which could be operationalised in an interprofessional manner to assist in countering the effects of the hidden curriculum. This should be culturally appropriate, fit for purpose and usable across disciplines. In addition to curriculum development, it is anticipated that professional staff development would also be needed. Both students and staff should use critical self-reflection when communicating with patients. Continuous self-development in intra- and interpersonal communication ${ }^{[7]}$ is important, especially given the negative behaviours, as described in the study.

\section{Study limitations}

As a case study with a limited number of participants in one medical school, the findings are not generalisable, although some of the recommendations may be applicable in other regions of SA with similar contexts. The findings should generate discussion and add to the body of knowledge on teaching communication to SA medical students and other health professionals.

\section{Conclusion}

Language and culture are important in SA healthcare, and it is imperative that attempts to revise curricula and improve communication in the SA context include both intercultural communication and provision for language needs of patients. In addition to efforts at institutional level, emphasis must also be placed on core professional competencies for students, and on developing the necessary reflexivity for them to practise successfully in the multilingual and multicultural society of SA.

Further research in the SA context is warranted for consensus on communication teaching and learning. Research should include students themselves - to promote learner-centredness; representatives of health professionals - to improve collaboration and for standardisation; and community members - for course designers to understand patients' challenges and priority needs for communication in healthcare.
Declaration. None.

Acknowledgements. The authors wish to acknowledge the dean of Teaching and Learning and the Research Office for assistance and funding, and participants in the study for their contributions.

Author contributions. Both authors are responsible for the content and writing of the article.

Funding. Funding for the study was provided by the Research Office of the University of KwaZulu-Natal, Durban, SA.

\section{Conflicts of interest. None.}

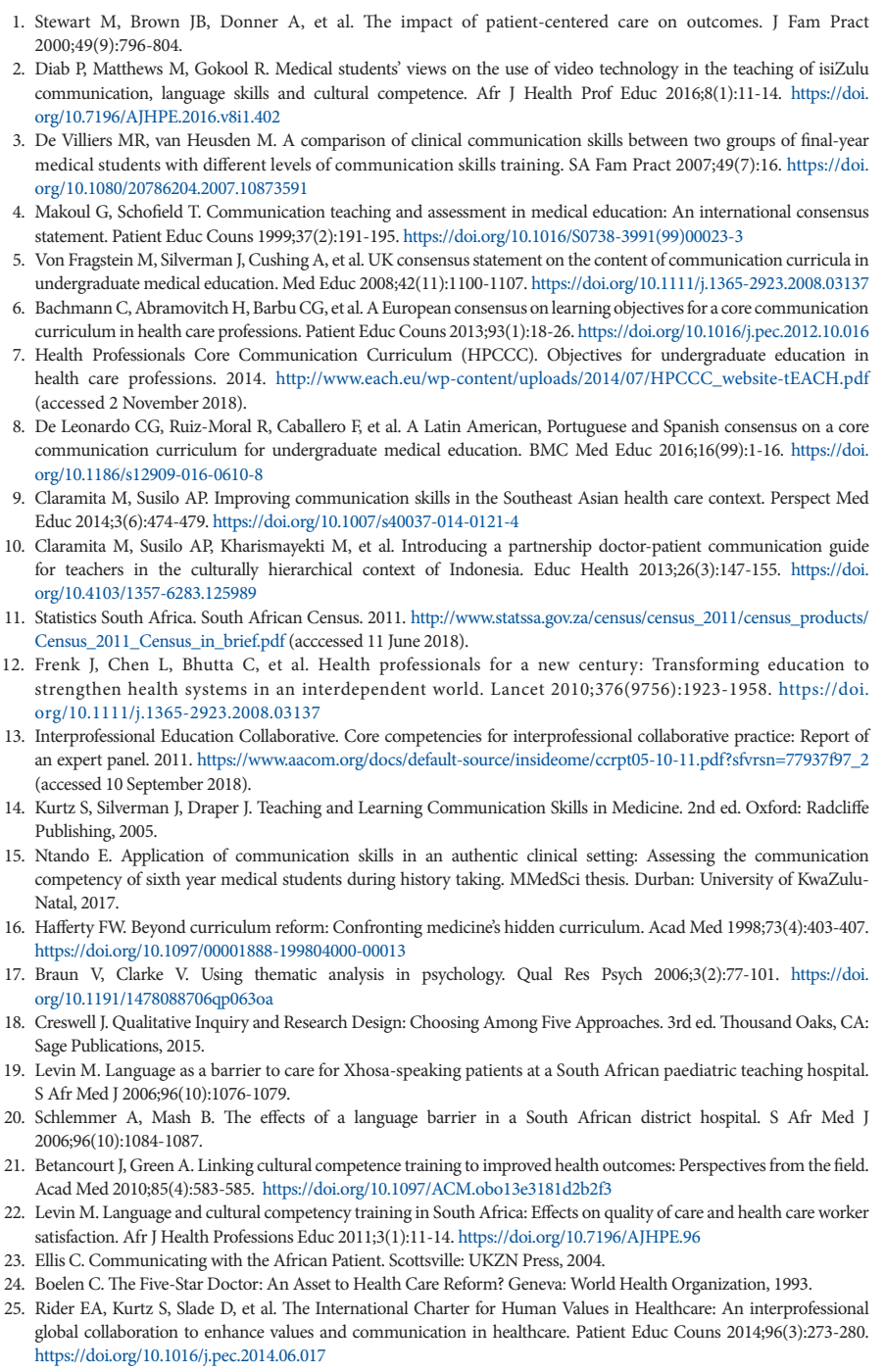

